Factors associated with retention among FSWs in a sex workers outreach program in Kenya

\title{
Factors associated with retention in HIV prevention and treatment clinical services among female sex workers enrolled in a sex workers' outreach program (SWOP) in Nairobi, Kenya.
}

\begin{abstract}
Female sex workers (FSWs) are among the key populations (KP) prioritized for comprehensive HIV programming in Kenya. Retention in the program is critical for prevention of HIV acquisition and transmission among FSWs and their sexual partners. We conducted a retrospective cohort analysis of data collected from FSWs enrolled between October 2016 and September 2017 at seven drop-in centers (DICs) in Nairobi, Kenya, to examine retention in HIV prevention and treatment services and identify the associated factors. We found a 3- and 12month retention of $24 \%$ and $17 \%$, respectively. FSWs aged $20-34$ years old were less likely to be retained compared to those 50 years or older. FSWs enrolled in a DIC located in their sub-county of residence or reporting ever using HIV pre- or post-exposure prophylaxis were more likely to be retained. Identifying predictors of FSWs disengagement from HIV services can inform the design and implementation of retention-enabling interventions.
\end{abstract}

Key words: female sex workers, retention, key populations. 


\section{BACKGROUND}

Kenya has a significant population of female sex workers (FSWs), estimated at 167,940, who are disproportionately affected by HIV in comparison to the general population [1][2]. Globally, $62 \%$ of new adult HIV infections are among KP and their sexual partners with sex workers accounting for $8 \%$, while in Eastern and Southern Africa, $28 \%$ of new adult HIV infections are among KP and their sexual partners with sex workers accounting for 5\% [3]. In addition, FSWs were 13-times more likely to have HIV-infection compared to other women of reproductive age [4]. In Kenya, approximately $33 \%$ of new HIV infections are attributable to KP with FSWs and their clients contributing $14.1 \%$ [5]. The HIV prevalence among FSWs (45.1\%) is about six times that of women in the general population (7.72\%) [6]. Furthermore, FSWs have an increased risk of disengagement from HIV services due to structural and individual barriers including HIV related stigma and discrimination, high mobility, and mental health conditions [7].

The World Health Organization (WHO) recommends the implementation of comprehensive health sector interventions with strategies fostering an enabling environment for KP [2]. In Kenya, the Ministry of Health (MOH) launched an HIV/Sexually Transmitted Infections (STI) prevention, care, and treatment program for KP, under the National AIDS and STI Control Program (NASCOP) in 2009, with the national guidelines recommending implementation of a combination of behavioral, structural, and biomedical interventions [8]. Some of the recommendations under the guidelines include HIV testing every three months with routine follow-up for education, distribution of condoms, pre-exposure prophylaxis (PrEP) eligibility/ risk assessment and monitoring, STI screening and treatment [8][9]. Regular and repeated HIV 
testing offered to FSWs contributes to early HIV case identification and linkage to treatment which, with good adherence and retention, results in HIV viral suppression reducing AIDSrelated illnesses or death and prevents further transmission of HIV (secondary prevention) [7][10]. The benefit of HIV biomedical prevention interventions such as PrEP, targeting highrisk FSWs who test HIV negative, is highly dependent on the user's adherence [11] necessitating close longitudinal monitoring.

Despite NASCOP's recommendation, limited data is available on retention of FSWs in HIV prevention and treatment programs. Due to the critical importance of the continuum of care for both HIV positive and negative FSWs, we examined the factors associated with retention in HIV preventive and treatment services among FSWs across seven KP-dedicated clinics in Nairobi, Kenya.

\section{METHODS}

\section{Study design and setting}

We conducted a retrospective cohort analysis of data from FSWs seeking care at the seven Sex Workers Outreach Program (SWOP) Drop-in Centers (DICs) located in Nairobi - Kenya. SWOP DICs are supported by the University of Manitoba (UoM) and the University of Maryland, Baltimore (UMB) in collaboration with Nairobi Metropolitan Services, Directorate of Health (DoH). The DoH and NASCOP provided oversight on program implementation. 


\section{Population}

All FSWs aged 18 years and over who enrolled in the program between October 2016 and September 2017 were included. In Kenya, sex workers are defined as adults aged 18 years and older who receive money or goods in exchange for sexual services, either regularly or occasionally [8]. FSWs who transferred-in from other sex workers' programs within the specified period were excluded for this analysis.

\section{Sampling}

We conducted systematic sampling of 3,774 FSWs newly enrolled at the seven DICs between October 2016 and September 2017. Following the Kenya Health Quality Improvement Framework (KHQIF), a sample size table was used to determine the number of clients that needed to be sampled at each DIC, based on its population size, to achieve a $95 \%$ representative sample [11]. A sampling frame was established for determination of the $n t h$ record for chart abstraction by dividing the DICs total number of clients enrolled by the site's determined sample size [12].

\section{Interventions}

The DICSs provide comprehensive HIV prevention and treatment services to KP including men who have sex with men (MSM), transgender women (TG), and FSWs. The program implements a hotspots-based peer-led model for clinical services delivery at the DICs and community locations through integrated clinical outreaches at KP hotspots such as streets, bars (with and without lodging), and brothels. The clinical services offered include biomedical and behavioral interventions (Table I). The core biomedical interventions include HIV testing services (HTS), syndromic screening for and management of STIs, HIV care and treatment, condoms and 
lubricant distribution, and screening for and management of tuberculosis (TB), gender-based violence (GBV), alcohol and drug use. The behavioral interventions include peer education, targeted communication for KP, and risk reduction counselling. Structural intervention, such as advocacy and community outreach to reduce stigma and discrimination using a human rightsbased approach, are also provided.

The program employs program officers, clinicians, lay counsellors, and peers who provide services both at the facility and community levels to address the health needs of the targeted clients. Peer leaders are identified through a participatory community approach, trained on peer mobilization and education using a standardized and approved NASCOP - MOH curriculum, and conduct their activities through outreach to their peers. Under the supervision of program officers, the peer educator maintains regular contact with their assigned FSW peer network enrolled in the program, offers risk-reduction counselling, and facilitates linkage to clinical and non-clinical services (e.g., legal support, economic empowerment resources). These activities are tracked using a peer calendar which is submitted monthly for review by the program officers.

The continuum of services upon enrollment into the program include risk and vulnerability screening, HTS, STI screening and syndromic management, anti-retroviral treatment (ART) initiation among HIV-infected clients, and individualized preventive interventions, such as PrEP, based on FSWs' risk profile. All services provided are documented in MOH-prescribed enrollment forms at enrollment and clinic visit forms at subsequent/ follow up visits. HIV negative FSWs are encouraged to screen for HIV every three months as per the Ministry of Health guidelines 


\section{Data sources and data collection}

Data for this evaluation was abstracted from the $\mathrm{MOH}$ approved enrollment and clinic visit forms in the client's medical records. Trained data clerks abstracted data from electronic medical record (EMR) and paper-based forms into the Kobo toolbox [13], an electronic data collection tool, with data validation incorporated into the data collection forms.

\section{Outcomes}

Outcomes of interest, aligned to recommended quarterly follow-up revisits [8], included shortterm (3-month) retention in the HIV prevention and treatment program defined as at least one visit between two and four months after enrollment, and long-term (12-month) retention defined as two or more clinical revisits including the last appointment within 10 to 12 months of followup. Our definition of retention was informed by the recommendation for quarterly interaction with KPs for provision of key services including HIV testing and STI screening among others [8].

\section{Independent variables}

We included socio-demographic variables (age, education, marital status, number of children, residence area), health-related information (HIV infection and care status, pregnancy status, contraception use, self-reported STI, use of PrEP or post-exposure prophylaxis (PEP), exposure to GBV, DIC- location) and behavioral variables (number of years in sex work, type of sex work, number of sex acts per week, use of condoms, use of alcohol and recreational drugs). Type of sex work was defined as venue-based if client solicitation happened within a building such as bars with and without lodging, casino, lodging or guesthouse, massage parlors, sex den /brothels, and non-venue if solicitation happened in open spaces such as streets and highways. 


\section{Statistical methods}

Descriptive statistics were reported for all the collected variables. Continuous variables were summarized using medians and inter-quartile range. Categorical variables were summarized using proportions. Chi square and Kruskal-Wallis tests were used to compare groups. Bivariate and multivariate logistic regression models were performed to analyze the outcomes. We used Generalized Estimating Equations (GEE) to control for clustering by DICs. For the final analysis, FSWs with unknown HIV status were excluded. For the multivariable models, we included variables that were statistically significant in the bivariate analysis at $\mathrm{p}<0.05$ or if they had been identified as important covariates in the literature. Data was analyzed using SAS 9.4 (Carey, NC).

\section{Ethics}

The Kenyatta National Hospital, University of Nairobi and the Human Research Ethics Committee at the University of Maryland Baltimore approved this evaluation. This project was also reviewed in accordance with the US Centers for Disease Control and Prevention (CDC) human research protection procedures and was determined to be research, but CDC investigators did not interact with human subjects or have access to identifiable data or specimens for research purposes.

\section{RESULTS}

\section{Baseline characteristics}

Of the 899 sampled cisgender- FSWs drawn from the seven DICs, 774 (86.1\%) were HIV uninfected, 78 (8.7\%) were HIV infected (44 known and 34 newly diagnosed at enrollment). HIV testing information was missing for 47 (5.2\%) at the time of enrollment. Of the 78 identified 
to be living with HIV at enrollment, $11(14.1 \%)$ were not enrolled in treatment, while 35 (44.9\%) and $32(41 \%)$ were enrolled at DIC and non DIC respectively.

At the time of enrolment, overall the majority (68.3\%) were aged between 20 and 34 years old, $58 \%$ were single and $52 \%$ had only completed primary school. The median age at enrolment was $30(\mathrm{IQR}=24-35)$ years old. Overall, 5\% had used PrEP within the review period. Two percent also reported having experienced GBV in the three months prior to enrollment. Fifty-eight percent had 2-4 years working as sex workers and 88\% were venue-based FSWs. While 90\% reported using a condom during the last sexual encounter, there was variation in their consistent use of condom; at $75.7 \%$ and $77.2 \%$ for regular and casual (non-regular) clients respectively, and $32.6 \%$ with a boyfriend. A total of $225(25 \%)$ reported using recreational drugs including alcohol, marijuana, cigarettes and Khat, while 321 (35.7\%) reported never having sex under the influence of alcohol (Table II).

In comparison to HIV uninfected women, HIV positive FSW were older (median 34 (IQR=2941) vs $30(\mathrm{IQR}=23-35))$, less educated (26.9\% vs. $15.4 \%$ had less than primary education), more divorced/separated (41.0\% vs. $36.8 \%$ ), had been on sex work for longer (53.8\% vs. $24.7 \%$ more than 4 years) and reported more GBV (5.1\% vs. $1.9 \%)$. Their condom used at last encounter were similar on both groups (91.0\% vs. $91.1 \%)$; however, HIV positive FSW have a higher frequency of condom use with their boyfriend (52.4\% vs. $21.4 \%$ reporting always) (Table II).

\section{Retention}

Overall, 860 (96\%) of the sampled enrollees, had at least one clinical revisit with a median of 29 (inter quartile range [IQR] 9-92) days to the first visit after enrollment; however, only 212 (24\%) of the sampled FSWs made a revisit to the clinic between 2-4 months after enrollment (shortterm retention), and 155 (17\%) attended at least two revisits after enrollment, with the last 
appointment between 10-12 months after enrollment (long-term retention). Retention differed by HIV status, short-term retention was $14.1 \%$ for HIV positive and $23.4 \%$ for HIV negative ( $\mathrm{p}=0.062$ ), while long-term retention was $28.2 \%$ for HIV positive FSW and $15.9 \%$ for HIV negative $(\mathrm{p}=0.005)$.

Short-term retention differed by DIC $(\mathrm{p}<0.001)$; therefore, further analysis was adjusted for clustering by DICs to avoid confounding. Factors associated with the short-term retention included: duration in sex work, sex worker residence in relation to DIC location, sex work venue, and practice of sex work under alcohol and drug influence. FSWs working for more than four years had twice the odds of being retained compared to those with less than two years of work (odds ratio [OR] 2.08, 95\%, confidence interval [CI]: 1.16-3.73). FSWs living in the same subcounty as the DICs where they enrolled had nearly three times higher odds of being retained (OR 2.74, 95\% CI: 1.11-6.72) compared with those living in a different sub-county. FSWs working on streets or highways were more likely to be retained (OR 0.61, 95\%, CI: 0.38-0.97) than those working at a venue including bars, brothels, and massage parlors. Women who self-reported to have sex always under the influence of alcohol were more likely to be retained (OR 1.79, 95\%, CI: 1.04-3.07) than those who never did. Similarly, FSWs who self-reported ever using drugs, besides alcohol, were more likely to be retained (OR 1.42, 95\%, CI: 1.16-1.75). All these variables remained significant after controlling for other confounders (Table III). Due to the small number of HIV positive clients in the cohort, further analysis was not stratified by HIV status. However, we also ran the logistic models after excluding HIV positives from the cohort. No significant change in the direction of the association was observed across the included factors in the multivariate model. Nevertheless, education became a significant factor for this HIV negative only cohort, where more education was associated with lower odds of short-term 
retention. Additionally, residing in the same county and use of drugs lost significance in the multivariate model.

Long-term retention also differed by DIC $(\mathrm{p}<0.001)$; therefore, further analysis was adjusted for clustering by DICs. FSWs who were HIV infected had twice the odds of being retained for one year in the program (OR 2.00, 95\%, CI: 1.09-3.64). FSWs aged 50 years or older had higher odds for retention in the first year in comparison to younger population 20-34 years of age (Table IV). FSWs living in the same sub-county with the DIC where they enrolled had higher odds of being retained (OR 1.64, 95\%, CI: 1.11-2.41) compared with those living in a different subcounty. FSWs who self-reported, at enrollment, ever having an STI had $42 \%$ higher odds of retention (OR 1.42, 95\%, CI: 1.03-1.96). Similarly, those who had ever used PrEP had higher odds of retention within a year of enrollment (PrEP OR 4.76, 95\%, CI: 1.98-11.43, PEP OR 1.72, 95\%CI: 1.01-2.95) (Table IV). Women who were pregnant at enrollment were less likely to be retained at 12 months after enrollment (OR 0.33, 95CI\%: 0.11-0.94). Similar to short-term retention, when restricting the analysis to only HIV negative FSWs, no significant change in direction of the association was observed across the included factors in the multivariate model. However, only age at enrollment, having ever a STI ever, and ever using PrEP or PEP remained significant in this model, potentially due to the smaller sample size (Table SII).

\section{DISCUSSION}

In this evaluation, we found low retention of FSWs in HIV prevention and treatment clinical services at SWOP DICs within the first 12 months of enrollment in Nairobi, Kenya. MoralesMiranda et al. reported retention at 12 months ranging from $7.7 \%$ to $42.7 \%$ in a study conducted in Guatemala, however, they defined retention as one or more follow up visits within 12 months 
of the initial visit [14]. Using a similar definition would have increased our retention to $96 \%$ highlighting the need for a standardized definition for retention in KP HIV programming.

In the adjusted analysis, we found the number of years in sex work (over 4 years) was positively associated with early retention, and women aged 50 years and above associated with higher 12month retention in comparison to women aged 20-34 years. Female sex workers living in the same sub-county as the DIC of enrollment were more likely to be retained at 3 and 12 months of enrollment, highlighting the need to locate DICs in close proximity to sex workers for ease of access and retention in HIV preventive and treatment services. Engaging in sex work under the influence of alcohol and use of other drugs were also positively associated with retention. We hypothesize that FSW that reported high-risk behaviors such as alcohol drinking and use of drugs may have received more intensive and frequent peer support. Further investigation is needed to better understand if the intensity of peer-support among FSWs is determined by assessed individual HIV risk behaviors including hazardous alcohol and substance use.

A positive HIV status was associated with better retention at 12 months. Our results differed from the study in Guatemala which found that positive HIV diagnosis negatively impacted retention [14]. This difference may be attributable to prompt ART start and desire to continue HIV care by the FSW as individuals who were not started on ART immediately following HIV diagnosis risk being lost to follow up. There was also a difference in the service delivery models as the DICs in this evaluation engaged peers as case managers to deliver ongoing support and counseling and community follow-up to HIV-infected FSWs. The study found that clients receiving PrEP were also likely to be retained in the long-term. The association of PrEP usage with higher retention, may be attributed to this intense peer support and follow-up and mandatory quarterly HTS and PrEP medication pick-up. Similar to Miranda-Morales et al., we 
found that sex work location, use of drugs and level of education did not significantly impact retention [14].

HIV positivity among our study population of new enrollees was $8.7 \%$, more than two-fold higher than HIV prevalence among the adults in Nairobi county [15]. However, other studies have reported higher HIV prevalence among FSWs in Kenya, varying from $29.5 \%$ to $56.5 \%$ [16] [17]. Differences in the study population may explain these divergences. In our evaluation, we included a sample of FSWs who sought health services voluntarily in a clinic while other studies may recruit FSWs using different recruitment strategies, including respondent-driven sampling methods, commonly used to reach marginalized populations that may not be accessing HIV services. The evaluation period coincides with the early phase of PrEP rollout in Kenya, which may explain the low uptake of PrEP (PrEP demonstration project conducted in 3/7 DICs in 2015 and national rollout in 2017).

Limitations of this evaluation include our definition of retention which was limited to attendance to the DICs and may not have completely captured community interactions with the FSWs through outreach activities. However, clinical services including HTS provided during outreaches from the DICs were updated in the clinical records. We may have further underestimated retention, by excluding data of FSWs who may have accessed HIV services at other DICs or in public health facilities. We were unable to examine factors associated with retention disaggregated by HIV status due to the small number of FSWs living with HIV in this cohort. The clinical and behavioral characteristics were self-reported by the FSWs which may have introduced bias due to social desirability or recall bias [18].

FSW retention in HIV prevention and treatment program is suboptimal according to NASCOP guidance. Our results suggest the urgent need to design, implement and evaluate client-centered 
Factors associated with retention among FSWs in a sex workers outreach program in Kenya

services enhancing HIV care continuum among FSWs. Implementation research is needed to gain additional understanding on the health services needs and preferences among FSWs to optimize retention for this population. Additional resources and/or investments will be needed to safeguard and ensure sustainability of KP programming in controlling the HIV epidemic. 
Factors associated with retention among FSWs in a sex workers outreach program in Kenya

\section{ACKNOWLEDGEMENT}

This study was supported by the U.S. President's Emergency Plan for AIDS Relief (PEPFAR) through the US Centers for Disease Control and Prevention (CDC) under the terms of cooperative agreement NU2GGH001962-02. The findings and conclusions in this report are solely those of the authors and do not necessarily represent the official position of the funding agencies. 


\section{REFERENCES}

1. National AIDS and STI Control Programme (NASCOP). Key Population Mapping and Size Estimation in Selected Counties in Kenya: Phase 1. 2019;(April).

2. WHO consolidated guidelines. HIV prevention, diagnosis, treatment and care for key populations. Published online 2016.

3. UNAIDS. Data 2020. Program HIV/AIDS. 2020:1-248. https://www.unaids.org/en/resources/documents/2020/unaidsdata\%0Ahttp://www.unaids.org/sites/default/files/media_asset/20170720_Data_book_201 7_en.pdf.

4. UNAIDS 2019. Global AIDS Update 2018 Miles To Go: Closing Gaps Breaking Barriers Righting Injustices. Unaids. Published online 2018:http://www.unaids.org/sites/default/files/media_as.doi:10.1111/j.16006143.2011.03542.x

5. National AIDS Control Council K. Kenya HIV Prevention Response and Modes of Transmission Analysis.; 2009.

6. Baral S, Beyrer C, Muessig K, et al. Burden of HIV among female sex workers in lowincome and middle-income countries : a systematic review and meta-analysis. Lancet Infect Dis. 2012;12(7):538-549. doi:10.1016/S1473-3099(12)70066-X

7. Lancaster KE, Cernigliaro D, Zulliger R, et al. HIV care and treatment experiences among female sex workers living with HIV in sub-Saharan Africa: A systematic review. African J AIDS Res. 2016;15(4):377-386. doi:10.2989/16085906.2016.1255652

8. National AIDS and STI Control Program K. National Guidelines for HIV / STI Programming for Key Population; 2014.

9. National AIDS \& STI Control Programme (NASCOP). Pre-exposure Prophylaxis for the Prevention of HIV Infection - A Toolkit for Health Service Providers. Published online 2017:57.

10. Myron S. Cohen M, Ying Q. Chen P., Marybeth McCauley MP., et al. Prevention of HIV1 Infection with Early Antiretroviral Therapy. N Engl J Med. 2011;365(6):493-505.

11. Dimitrov DT, Mâsse BR, Donnell D. PrEP adherence patterns strongly affect individual HIV risk and observed efficacy in randomized clinical trials. J Acquir Immune Defic Syndr. 2016;72(4):444-451. doi:10.1097/QAI.0000000000000993

12. Kenya Ministry of Health. KHQIF Operational Manual.; 2014.

13. Kobo Tool Box. 2019;(Version 2.019.07 March 2019).

https://kobo.humanitarianresponse.info 
14. Morales-Miranda S, Jacobson JO, Loya-Montiel I, et al. Scale-up, retention and HIV/STI prevalence trends among female sex workers attending VICITS clinics in Guatemala. PLoS One. 2014;9(8):1-9. doi:10.1371/journal.pone.0103455

15. National AIDS and STI Control Programme (NASCOP). Preliminary KENPHIA 2018 Report. Nairobi: NASCOP; 2020. Published online 2020.

16. Musyoki, H., Kellogg, T.A., Geibel S et al. Prevalence of HIV, STIs, and risk behaviors among FSWs in Nairobi, Kenya. AIDS Behav. 2016;19(Suppl 1). doi:10.1007/s10461014-0919-4.Prevalence

17. Vandenhoudt HM, Langat L, Menten J, et al. Prevalence of HIV and Other Sexually Transmitted Infections among Female Sex Workers in Kisumu, Western Kenya, 1997 and 2008. PLoS One. 2013;8(1):1-15. doi:10.1371/journal.pone.0054953

18. Althubaiti A. Information bias in health research: Definition, pitfalls, and adjustment methods. J Multidiscip Healthc. 2016;9:211-217. doi:10.2147/JMDH.S104807 